\title{
Aceptación diferenciada de alimentos transgénicos de origen vegetal y animal en la Región de La Araucanía, Chile
}

\author{
Berta Schnettler, Oriana Sepúlveda y Danilo Ruiz \\ Departamento de Producción Agropecuaria, \\ Universidad de La Frontera. Casilla 54-D. Temuco, Chile
}

\begin{abstract}
B. Schnettler, O. Sepúlveda, and D. Ruiz. 2008. Differentiated acceptance of transgenic foods of animal and vegetable origin in the Region of La Araucania, Chile. Cien. Inv. Agr. 35(2):169-180. Genetically modified transgenic foods (TF) have generated considerable debate in developed countries. Therefore, a survey of 400 people was conducted to assess the importance of this variable in consumer decisions regarding the purchase of liquid milk and tomato sauce in Temuco (IX ${ }^{\text {th }}$ Region, Chile). This survey also evaluated the existence of different market segments. Using a conjoint analysis, we established that the presence of genetic modification (GM) in food was a more important factor than either the brand or the price in both types of food. Using a cluster analysis, five consumer groups were distinguished, with two large groups representing $46.8 \%$ and $18.8 \%$ of the total number of people surveyed. These two groups felt the existence of GMs to be important, but only the largest group preferred the alternatives with no GMs. For the third group (16.8\%), brand was the most important attribute, but a preference for non-TF was also shown. The fourth group (10.5\%) placed great importance on price and preferred non-TF. The smallest group (7.3\%) placed a slightly higher value on the price of milk and on the brand of tomato sauce, showing a preference for TF. The five groups all rejected the store-brand product and most consumers reacted positively to lower prices. Therefore, with the results of this investigation, it can be concluded that the absence of GMs in milk and tomato sauce is a desirable condition for a large proportion of consumers in Temuco (74\%), but there are groups of consumers (26\%) that accept genetic manipulation of these foods.
\end{abstract}

Key words: Conjoint analysis, cluster analysis, genetically modified foods, genetic engineering, marketing, transgenic food.

\section{Introducción}

Durante los últimos años, se ha desatado un conflicto en relación con los riesgos y beneficios del consumo de alimentos transgénicos (AT) para la salud humana y el medio ambiente. La denominación alimento transgénico se usa para alimentos con alguna materia prima derivada de un organismo genéticamente modificado, principalmente a través de transgenia, o constituido por el organismo genéticamente modificado propiamente tal (Anónimo 1, 2002).

Recibido 05 diciembre 2007. Aceptado 13 mayo 2008.

${ }^{1}$ Dirigir correspondencia a B. Schnettler: bschnett@ufro.cl
La primera generación de organismos modificados genéticamente (MG) estuvo enfocada en la incorporación de caracteres productivos que beneficiaran a los productores (ej. introducción de genes para resistencia a insectos y tolerancia a herbicidas), mientras la segunda generación incorporó nuevos caracteres con beneficios para el consumidor (ej. mayor aporte de vitaminas, antioxidantes).

Numerosos estudios dan cuenta de la preferencia del consumidor por alimentos libres de manipulación genética y rechazo hacia los ATs (Ganiere et al., 2006; Ho et al., 2006 Larue et al., 2004; Lusk et al., 2003; Lusk et al., 2001). No obstante, algunos consumidores pueden ser indiferentes a esta condición (Townsend, 
2006). La resistencia a la introducción de MG no es universal (Mucci y Hough, 2004). Las actitudes respecto a la biotecnología dependen del organismo en el cual se aplica y el tipo de modificación realizada. Las modificaciones genéticas en plantas o microorganismos son más aceptadas que las modificaciones en animales (Frewer et al., 2004). Además, los consumidores europeos se preocupan del consumo indirecto de este tipo de alimentos, es decir el uso de AT en alimentación animal (Lähteenmäki et al., 2003; Lusk et al., 2003; Roosen et al., 2003). Asimismo, es relativamente más aceptada una MG para reducir el uso de pesticidas que las MGs para caracteres que permitan reducir los costos de producción (Kaye-Blake et al., 2005) o, si la modificación además presenta beneficios para el consumidor (Hossain et al., 2003; Lähteenmäki et al., 2003).

La actitud del consumidor hacia los AT está determinada por los riesgos y beneficios percibidos, los que se encuentran determinados por el conocimiento que se tiene sobre los ATs, considerado aun bajo (Byrne, 2006; Townsend, 2006; Hossain et al., 2003; Verdume y Viaene, 2003). En Taiwán (Chen y Li, 2007) y en el Reino Unido (Costa-Font y Mossialos, 2007) se ha determinado que el mayor conocimiento sobre los ATs disminuye la percepción de riesgo de los consumidores, resultando en una actitud más positiva hacia los ATs. En forma concordante Ho et al. (2006) y Huang et al. (2006) determinaron que los consumidores chinos no tienen una posición clara frente al consumo de ATs por falta de conocimiento. Beneficios sustanciales, como un menor precio (Kaye-Blake et al., 2005; Jaeger et al., 2004; Noussair et al., 2004; Hossain et al., 2003; Lusk et al., 2002), mayor valor nutritivo (KayeBlake et al., 2005; Lähteenmäki et al., 2003), beneficios para la salud o el medio ambiente (Ganiere et al., 2006; Kaye-Blake et al., 2005; Mucci y Hough; 2004; Napier et al., 2004; Lähteenmäki et al., 2003) o un mejor sabor (Hossain et al., 2003; Lähteenmäki et al., 2003), compensarían el riesgo percibido resultando en una actitud positiva hacia los ATs.

Los consumidores tienen mejor disposición para comprar AT de marcas conocidas respecto de alimentos genéricos debido a la confianza que les brindan las marcas conocidas (Finucane y Holup, 2005; Verdume y Viaene, 2003). Por otra parte, según algunas investigaciones existe mayor disposición a adquirir alimentos transgénicos en hombres y personas de mediana edad (Napier et al., 2004; Hossain et al., 2003; Verdume y Viaene, 2003), mayor rechazo en personas mayores, con menor nivel educacional (Ganiere et al., 2006; Baker y Burnham; 2002) de estilo de vida liberal (Hossain et al., 2003). Sin embargo, existen investigaciones señalando que la aceptación de los AT es independiente de las características sociodemográficas (Hossain y Onyango, 2004; Lähteenmäki et al., 2003; Lusk et al., 2001).

En América del Sur existen escasos estudios sobre la aceptabilidad de ATs. En Brasil, Oda y Soares (2000) determinaron que 53\% de los encuestados había escuchado hablar de biotecnología, mientras la disposición a comprar AT se correlacionó con el lugar de residencia, ingreso y educación. En Argentina e independientemente del género del encuestado, Mucci y Hough (2004) determinaron mayor aceptación hacia AT que beneficien la salud o el medio ambiente respecto de AT de menores precios. En Santiago, Chile, Gil et al. (2001) determinaron un escaso conocimiento de la biotecnología y de los AT, existiendo una negativa percepción pública del uso de alimentos transgénicos.

En Chile, la Resolución No 1927 de 1993 sobre Normas y Regulación de Liberación de Transgénicos (Servicio Agrícola y Ganadero, Ministerio de Agricultura) actualizada por la $\mathrm{N}^{\circ} 1523$ de 2001, autoriza la entrada de semillas transgénicas para multiplicación con fines de exportación. No obstante, Chile está actualmente importando AT, maíz y soya de Argentina y EUA. Se desconoce la cantidad de transgénicos que ingresan, pues los granos llegan mezclados con no transgénicos y los productos elaborados no son debidamente etiquetados (Ríos, 2005). Al respecto, estudios recientes en Argentina, EUA y Australia señalan la importancia de informar al consumidor sobre el uso de ingredientes $\mathrm{MG}$ en el etiquetado de los alimentos (Anderson y Jackson, 2005; Mucci y Hough, 2004; Napier et al., 2004). 
En función de estos antecedentes, el objetivo de este estudio fue diferenciar segmentos de consumidores en Temuco, capital de la Región de La Araucanía (IX Región), Chile, en relación con la importancia relativa de atributos relevantes del producto (precio, marca) y la condición de transgénico en la decisión de compra de alimentos de origen vegetal y animal.

\section{Materiales y métodos}

\section{Encuesta}

Se realizó una encuesta personal a una muestra de 400 consumidores mayores de 18 años de Temuco ( $38^{\circ} 45^{\prime}$ lat. $\mathrm{S}, 73^{\circ} 03^{\prime}$ long. O). El número de encuestados se obtuvo mediante la fórmula de muestreo aleatorio simple para poblaciones no finitas ( $\mathrm{N}>100.000$; Temuco: 245.347 habitantes al Censo de 2002), considerando 95\% de confianza y $5 \%$ de error de estimación con p y q de 0,5 (Fernández, 2002).

Se usó un cuestionario con preguntas cerradas para determinar si el encuestado ha recibido información de AT, si conoce su significado y frecuencia de lectura de las etiquetas de los alimentos que compra. Luego se les leyó la siguiente definición de AT: "los alimentos transgénicos son aquellos a los cuales se les ha introducido en forma artificial un gen foráneo a nivel de embrión, de modo que al reproducirse mantengan esta nueva característica, por ejemplo para eliminar el uso de pesticidas, fungicidas y herbicidas durante su cultivo".

Posteriormente, se consultó si consideran necesario que el etiquetado indique que el alimento contiene ingredientes MG. Se incluyeron preguntas de clasificación: género, edad, tamaño del grupo familiar, presencia de hijos y su edad, estilo de vida, ocupación y estudios del jefe de hogar y, la tenencia de diez bienes domésticos. Estas dos últimas variables se incluyeron para determinar el grupo socioeconómico correspondiente, clasificado como $\mathrm{ABC} 1$ (alto y medio alto), $\mathrm{C} 2$ (mediomedio), C3 (medio-bajo), D (bajo) y E (muy bajo) (Anónimo 2, 2004).

La encuesta se aplicó en dos supermercados de Temuco entre abril y junio de 2006, luego de validar el cuestionario mediante una prueba preliminar con el $10 \%$ de la muestra a encuestar.

\section{Análisis estadísticos}

Se utilizó análisis conjunto para determinar la importancia de la condición de transgénico de un alimento en la decisión de compra. Este análisis permitió estimar la importancia relativa de los atributos de un producto y estimar valores de utilidad o preferencia para cada nivel de un atributo. Las utilidades estimadas indicaron el grado de preferencia o rechazo por cada nivel de cada atributo (Hair et al., 1999). Este análisis se realizó para determinar la importancia de los siguientes atributos y niveles: 1 . Presencia o ausencia de MG (transgénico y no transgénico), 2. Marcas (nacionales y propias), 3. Precios en la compra de leche líquida y salsa de tomate.

Las marcas propias fueron marcas creadas, controladas $y / 0$ vendidas por minoristas. Las marcas nacionales o de fabricante (Nestlé y Malloa en leche y salsa de tomate, respectivamente) correspondieron a productos vendidos por el fabricante a través de minoristas (Sethuraman y Cole, 1999). Los niveles de precio fueron $\$ 400 \pm 40$ el litro leche líquida y $\$ 165 \pm 15$ el envase de $200 \mathrm{~g}$ de salsa de tomate, establecidos según el precio promedio en Temuco al momento de realizar la encuesta. A partir de estos atributos y niveles se obtuvo ocho combinaciones $(2 \times 2 \times 2$, presencia o ausencia de MG x marca x precio) en cada alimento. La función de preferencia correspondió al Modelo de Punto Ideal (Hair et al., 1999).

Los datos se obtuvieron por el procedimiento de perfil total, para lo cual se elaboraron ocho tarjetas con una especificación para cada atributo en ambos alimentos. Cada encuestado ordenó las tarjetas desde la más preferida a la menos preferida, usando una escala de 1 a 8 , donde $1=$ más preferida y $8=$ menos preferida. Para el atributo precio se estableció una relación lineal, debido a que generalmente a mayor precio la preferencia es menor. Los atributos restantes se consideraron como variables discretas. Para determinar la bondad de ajuste del modelo se utilizó correlación de Pearson y, se usó la Tau 
de Kendall para determinar si la ordenación de los estímulos estimada según la función de ordenación correspondió con la ordenación real del encuestado (Hair et al., 1999).

Para determinar segmentos de consumidores según la importancia y preferencia hacia la existencia de MGs en el alimento, marca y precio, se usó análisis de conglomerados jerárquicos, con el método de Ward como forma de encadenamiento y con la distancia euclídea al cuadrado como medida de similitud entre objetos (Hair et al., 1999). El número de conglomerados se obtuvo mediante observación del dendrograma y se confirmó con la determinación del porcentaje de cambio de los coeficientes de conglomeración recompuestos. Para describir los segmentos se aplicó la prueba de chi cuadrado $(\chi 2)$ de Pearson a las variables discretas. Se realizó análisis de varianza para establecer la significancia de las diferencias obtenidas entre las preferencias por los diferentes niveles de los distintos atributos. Los promedios de variables con diferencias significativas ( $\mathrm{p} \leq 0,05)$, se separaron según la prueba de comparación múltiple de Tukey (Lea et al., 1997). Se usó el programa SPSS 14.0 (SPSS.Inc., Chicago, EUA).

\section{Resultados}

En la muestra de consumidores encuestados hubo similar cantidad de hombres y mujeres, predominando consumidores entre 35 y 54 años, de familias con tres a cuatro integrantes, sin hijos o con hijos entre 13 y 17 años, agricultores y trabajadores independientes, con estilo de vida conservador y liberal, predominantemente de los grupos socioeconómicos más altos (Cuadro 1).

Aproximadamente, $60 \%$ de los encuestados indicó haber recibido información sobre AT, pero menos del 30\% sabía su significado. Respecto al etiquetado, fueron superiores las proporciones de consumidores que indicaron leer las etiquetas "generalmente" y "ocasionalmente", mientras la mayoría estuvo de acuerdo en que la etiqueta debe indicar el uso de ingredientes MG en la elaboración del alimento (Cuadro 1).
Mediante análisis conjunto se obtuvo en la muestra total que el factor de mayor importancia en la compra de ambos alimentos fue la presencia o ausencia de manipulación genética $(44,7 \%$ en leche y $44,6 \%$ en salsa de tomate), seguido por la marca $(29,8 \%$ en leche y $30,0 \%$ en salsa de tomate) y el precio ( $25,5 \%$ en leche y $25,4 \%$ en salsa de tomate). El consumidor experimentó preferencia hacia la leche y salsa de tomate producida sin MG y rechazo respecto a la presencia de $\mathrm{MG}$. Se obtuvo preferencia por las marcas nacionales y rechazo hacia la marca propia. Ambos niveles de precio reportaron rechazo, el que se incrementó (cifras de preferencia más negativas) al acceder a comprar los productos a un mayor precio indicando que no existe asociación entre precio y calidad (Cuadro 2). Los coeficientes de correlación de Pearson $(0,999$ en leche y 0,998 en salsa de tomate) y Tau de Kendall (1,000 en ambos alimentos) fueron cercanos o iguales a $1(\mathrm{p}=0,001)$.

Mediante análisis de conglomerados fue posible distinguir significativamente $(\mathrm{p} \leq 0,05)$ cinco grupos de consumidores en la importancia y preferencia por la presencia o ausencia de MG, marca y precio en leche y salsa de tomate (Cuadro 2).

El grupo mayoritario (Grupo 2) que representó $(n=187)$ el $46,8 \%$ de la muestra de encuestados otorgó mayor importancia a la presencia o ausencia de MG en ambos alimentos (58,8\% en leche y $56,9 \%$ en salsa de tomate), estadísticamente similar al Grupo 4 y superior al resto de los segmentos ( $\mathrm{p} \leq 0,05)$. El valor positivo de preferencia hacia leche $(1,804)$ y salsa de tomate $(1,866)$ sin $\mathrm{MG}$ indican preferencia por esta condición significativamente superior a los otros grupos $(\mathrm{p} \leq 0,05)$, mientras los valores negativos de preferencia frente a ambos alimentos transgénicos (-1,804 en leche y 1,866 en salsa de tomate), significativamente inferiores al resto de los grupos, evidenciaron un alto rechazo hacia la MG en los alimentos. Este segmento asignó baja importancia a la marca $(21,99 \%)$, estadísticamente similar al Grupo 1 e inferior al resto de los grupos ( $\mathrm{p} \leq$ $0,05)$, y baja importancia al precio $(19,18 \%)$, estadísticamente similar al Grupo 4 e inferior a los otros grupos $(\mathrm{p} \leq 0,05)$. 
Cuadro 1. Descripción porcentual de la muestra de compradores encuestados habituales de supermercados de la ciudad de Temuco, Región de la Araucanía, Chile. Junio de 2006.

Table 1. Description in percentage of the sample of habitual supermarket consumers in Temuco, IXth Region, Chile. June, 2006.

\begin{tabular}{|c|c|c|}
\hline Muestra de encuestados & Composición & $\begin{array}{c}\text { Muestra total } \\
(\mathrm{n}=400) \\
\%\end{array}$ \\
\hline \multirow[t]{2}{*}{ Género } & Femenino & 51,8 \\
\hline & Masculino & 48,2 \\
\hline \multirow[t]{3}{*}{ Edad } & $<$ de 35 años & 33,2 \\
\hline & 35-54 años & 45,3 \\
\hline & 55 años o más & 21,5 \\
\hline \multirow[t]{3}{*}{ Tamaño del grupo familiar } & 1-2 integrantes & 8,0 \\
\hline & 3-4 integrantes & 66,2 \\
\hline & 5 ó más & 25,8 \\
\hline \multirow[t]{5}{*}{ Presencia y edad hijos } & Sin hijos & 28,2 \\
\hline & Hijos $<5$ años & 9,0 \\
\hline & Hijos 5-12 años & 10,0 \\
\hline & Hijos 13-17 años & 30,3 \\
\hline & Hijos $\geq 18$ años & 22,5 \\
\hline \multirow[t]{7}{*}{ Ocupación } & Independiente & 22,0 \\
\hline & Empresario & 10,5 \\
\hline & Agricultor & 29,8 \\
\hline & Empleado particular & 19,3 \\
\hline & Empleado público & 12,5 \\
\hline & Jubilado & 2,3 \\
\hline & Cesante & 3,8 \\
\hline \multirow[t]{5}{*}{ Estilo de vida } & Conservador & 36,0 \\
\hline & Liberal & 36,2 \\
\hline & Vegetariano & 10,8 \\
\hline & Deportista & 9,2 \\
\hline & Innovador & 7,8 \\
\hline \multirow[t]{5}{*}{ Grupo socioeconómicol } & $\mathrm{ABC} 1$ & 34,8 \\
\hline & $\mathrm{C} 2$ & 33,0 \\
\hline & $\mathrm{C} 3$ & 17,2 \\
\hline & $\mathrm{D}$ & 12,8 \\
\hline & $\mathrm{E}$ & 2,2 \\
\hline \multirow[t]{2}{*}{ Ha recibido información de AT2 } & $\mathrm{Si}$ & 59,0 \\
\hline & No & 41,0 \\
\hline \multirow[t]{2}{*}{ Conoce el significado de la palabra AT2 } & $\mathrm{Si}$ & 26,3 \\
\hline & No & 73,7 \\
\hline \multirow{5}{*}{ Frecuencia de lectura etiquetas de los alimentos } & Siempre & 12,3 \\
\hline & Generalmente & 28,8 \\
\hline & Ocasionalmente & 31,3 \\
\hline & Casi nunca & 20,6 \\
\hline & Nunca & 7,0 \\
\hline \multirow[t]{2}{*}{ La etiqueta debe indicar la presencia de MG3 } & $\mathrm{Si}$ & 99,3 \\
\hline & No & 0,7 \\
\hline
\end{tabular}

\footnotetext{
${ }^{1}$ Grupos socioeconómico, ABC1 (alto y medio alto), C2 (medio-medio), C3 (medio-bajo), D (bajo) y E (muy bajo). ${ }^{2} \mathrm{AT}$, alimentos transgénicos. ${ }^{3} \mathrm{MG}$, modificación genética.

${ }^{1}$ Socioeconomic group, $\mathrm{ABCl}$ (high and middle-high), C2 (middle-middle), C3 (middle-lower), D (lower) y E (very low). ${ }^{2} T F$, transgenic foods. ${ }^{3} \mathrm{GM}$, genetic modification.
}

De acuerdo con estos resultados el Grupo 2 se puede denominar "Consumidores sensibles a la MG en los alimentos, rechazan los AT" (Cuadro 2). En relación con la muestra total, el Grupo 2 presentó superior proporción de personas menores de 35 años $(41,7 \%)$, familias con cinco o más integrantes $(32,1 \%)$, consumidores sin hijos $(40,1 \%)$, de estilo de vida conservador (44,9\%), mayor proporción de personas con conocimiento de los ATs $(31,0 \%)$ $\mathrm{y}$ que ocasionalmente lee las etiquetas de los alimentos (39,2\%) (Cuadro 3). 
Cuadro 2. Importancia de la manipulación genética (MG), marca y precio en el proceso de decisión de compra de leche líquida y salsa de tomate, y preferencias de los niveles de cada atributo de grupos obtenidos con análisis de conglomerados en la ciudad de Temuco, Región de La Araucanía, Chile. Junio de 2006.

Table 2. Importance of genetic manipulation $(M G)$, brand and price in the decision to purchase milk and tomato sauce, and utilities of the levels of attribute in the groups obtained by cluster analysis in Temuco, IXth Region, Chile. June, 2006.

\begin{tabular}{|c|c|c|c|c|c|c|c|c|}
\hline & $\begin{array}{l}\text { Muestra total } \\
n=400\end{array}$ & $\begin{array}{c}\text { Grupo } 1 \\
n=42\end{array}$ & $\begin{array}{c}\text { Grupo } 2 \\
n=187\end{array}$ & $\begin{array}{c}\text { Grupo } 3 \\
n=67\end{array}$ & $\begin{array}{c}\text { Grupo } 4 \\
n=75\end{array}$ & $\begin{array}{c}\text { Grupo } 5 \\
n=29\end{array}$ & $\mathrm{~F}$ & Sig. ${ }^{3}$ \\
\hline \multicolumn{9}{|c|}{ Importancia de los atributos en leche } \\
\hline Presencia/ausencia MG & 44,7 & $20,9 \mathrm{c}^{2}$ & $58,8 \mathrm{a}$ & $16,5 \mathrm{c}$ & $52,8 \mathrm{a}$ & $32,1 \mathrm{~b}$ & 233,55 & $* * *$ \\
\hline Marca & 29,8 & $19,8 \mathrm{c}$ & $22,0 \mathrm{c}$ & $57,5 \mathrm{a}$ & $29,5 \mathrm{~b}$ & $30,4 \mathrm{~b}$ & 119,68 & $* * *$ \\
\hline Precio & 25,5 & 59,3 a & $19,2 \mathrm{~d}$ & $26,0 \mathrm{c}$ & $17,7 \mathrm{~d}$ & $37,5 \mathrm{~b}$ & 139,04 & $* * *$ \\
\hline \multicolumn{9}{|c|}{ Preferencia por niveles de los atributos en lechel } \\
\hline Con manipulación genética & $-0,569$ & $-0,01 b^{2}$ & $-1,80 \mathrm{c}$ & $-0,03 \mathrm{~b}$ & $1,49 \mathrm{a}$ & $0,02 \mathrm{~b}$ & 222,73 & $* * *$ \\
\hline Sin manipulación genética & 0,569 & $0,01 \mathrm{~b}$ & $1,80 \mathrm{a}$ & $0,03 \mathrm{~b}$ & $-1,49 \mathrm{c}$ & $-0,02 \mathrm{~b}$ & 222,73 & $* * *$ \\
\hline Marca nacional & 0,777 & $0,52 \mathrm{bc}$ & $0,59 \mathrm{~b}$ & $1,88 \mathrm{a}$ & $0,68 \mathrm{~b}$ & $0,03 \mathrm{c}$ & 73,81 & $* * *$ \\
\hline Marca propia & $-0,777$ & $-0,52 a b$ & $-0,59 b$ & $-1,88 \mathrm{c}$ & $-0,68 b$ & $-0,03 \mathrm{a}$ & 73,81 & $* * *$ \\
\hline Precio $\$ 360 / \mathrm{L}$ & $-0,964$ & $-3,88 \mathrm{c}$ & $-1,10 b$ & $-0,18 \mathrm{a}$ & $0,05 \mathrm{a}$ & $-0,29 a$ & 72,76 & $* * *$ \\
\hline Precio $\$ 440 / \mathrm{L}$ & $-1,928$ & $-7,76 \mathrm{c}$ & $-2,20 b$ & $-0,36 \mathrm{a}$ & 0,11 a & $-0,59 a$ & 72,76 & $* * *$ \\
\hline \multicolumn{9}{|c|}{ Importancia de los atributos en salsa de tomate } \\
\hline Presencia/ausencia MG & 44,6 & $25,7 b^{2}$ & 56,9 a & $19,2 \mathrm{~b}$ & $57,0 \mathrm{a}$ & $23,1 \mathrm{~b}$ & 160,49 & $* * *$ \\
\hline Marca & 30,0 & $21,3 \mathrm{c}$ & $22,1 \mathrm{c}$ & 57,7 a & $25,7 \mathrm{c}$ & $39,8 \mathrm{~b}$ & 119,28 & $* * *$ \\
\hline Precio & 25,4 & 53,0 a & $21,0 \mathrm{c}$ & $23,1 \mathrm{c}$ & $17,3 \mathrm{c}$ & $37,1 \mathrm{~b}$ & 48,14 & $* * *$ \\
\hline \multicolumn{9}{|c|}{ Preferencia por niveles de los atributos en salsa de tomatel } \\
\hline Con manipulación genética & $-0,633$ & $-0,20 b^{2}$ & $-1,87 \mathrm{c}$ & $-0,06 \mathrm{~b}$ & $1,43 \mathrm{a}$ & $0,02 \mathrm{~b}$ & 259,08 & $* * *$ \\
\hline Sin manipulación genética & 0,633 & $0,20 \mathrm{~b}$ & $1,87 \mathrm{a}$ & $0,06 \mathrm{~b}$ & $-1,43 \mathrm{c}$ & $-0,02 \mathrm{~b}$ & 259,08 & $* * *$ \\
\hline Marca nacional & 0,748 & $0,61 \mathrm{~b}$ & $0,57 \mathrm{~b}$ & $1,85 \mathrm{a}$ & $0,54 \mathrm{~b}$ & $0,06 \mathrm{~b}$ & 60,41 & $* * *$ \\
\hline Marca propia & $-0,748$ & $-0,61$ a & $-0,57 \mathrm{a}$ & $-1,85 \mathrm{~b}$ & $-0,54 \mathrm{a}$ & $-0,06 \mathrm{a}$ & 60,41 & $* * *$ \\
\hline Precio $\$ 150 /$ envase $200 \mathrm{~g}$ & $-0,971$ & $-3,51 \mathrm{~d}$ & $-1,33 \mathrm{c}$ & $-0,22 b$ & $-0,17 \mathrm{~b}$ & $1,22 \mathrm{a}$ & 85,37 & $* * *$ \\
\hline Precio $\$ 180 /$ envase $200 \mathrm{~g}$ & $-1,942$ & $-7,02 \mathrm{~d}$ & $-2,66 c$ & $-0,45 \mathrm{~b}$ & $-0,33 \mathrm{~b}$ & 2,45 a & 85,37 & $* * *$ \\
\hline
\end{tabular}

${ }^{1}$ Cifras de preferencia en los distintos niveles de un atributo con signo negativo indican rechazo para el consumidor.

${ }^{2}$ Promedios seguidos por letras iguales en cada fila no son estadísticamente diferentes según la prueba de comparación múltiple de Tukey $(\mathrm{p}=0,05)$.

${ }^{3}$ Sig. = significativo; $* * *$, estadísticamente significativo a $\mathrm{p} \leq 0,001$.

${ }^{1}$ Utility numbers in the different levels from an attribute with a negative sign indicate utility loss for the consumer.

${ }^{2}$ Means followed by the same letters in each row are not statistically different according to the Tukey's multiple comparison test $(p=0.05)$.

${ }^{3}$ Sig. $=$ significant $; * * *$, statistically significant at $p<0.001$.

El segundo grupo (Grupo 4) en importancia numérica $(\mathrm{n}=75)$ representó el $18,8 \%$ de la muestra total de encuestados, también asignó mayor importancia a la presencia de MG $(52,8 \%$ en leche y $57,0 \%$ en salsa de tomate), fue estadísticamente similar al Grupo 2 y significativamente superior a los Grupos 1,3 y 5 ( $\mathrm{p} \leq 0,05)$. No obstante, a diferencia del Grupo 2 , en este segmento hubo rechazo hacia los alimentos sin MG (cifras de preferencia negativa de $-1,490$ en leche y $-1,426$ en salsa de tomate) y preferencia por los alimentos transgénicos (preferencia positiva de 1,490 en leche y 1,426 en salsa de tomate), significativamente superior a los Grupos 1, 2, 3 y 5 ( $\mathrm{p} \leq 0,05)$.
Este segmento asignó mediana importancia a la marca $(29,5 \%$ en leche y $25,7 \%$ en salsa de tomate) y baja importancia al precio (17,7 y 17,3\%; respectivamente), fue estadísticamente similar al Grupo 2 e inferior a los Grupos 1, 3 y 5 en leche, y estadísticamente similar a los Grupos 2 y 3 e inferior a los Grupos 1 y 5 en salsa de tomate $(\mathrm{p} \leq 0,05)$.

De acuerdo con estos resultados el Grupo 4 puede ser llamado "Consumidores sensibles a la MG en los alimentos, prefieren AT" (Cuadro 2). Respecto de la muestra total, este grupo presentó mayor proporción de personas entre 35 y 54 años $(54,7 \%)$, de familias con tres a 
cuatro integrantes $(82,7 \%)$, con hijos entre 13 y 17 años $(48,0 \%)$, de estilo de vida liberal $(41,3 \%)$ y que generalmente lee las etiquetas $(41,4 \%)$ (Cuadro 3).

El tercer grupo (Grupo 3) representó $(\mathrm{n}=67)$ el $16,8 \%$ de la muestra total de encuestados, y asignó mayor importancia a la marca de la leche y salsa de tomate con un 57,5 y 57,7\%, respectivamente, significativamente superior al resto de los segmentos $(p \leq 0,05)$. Fue el grupo que dio menor importancia a la existencia de MG, estadísticamente similar al Grupo 1 e inferior a los Grupos 2, 4 y 5 ( $\mathrm{p} \leq 0,05)$ en leche fluida $\mathrm{y}$, estadísticamente similar a los Grupos 1 y 5 pero inferior a los Grupos 2 y 4 en salsa de tomate (p $\leq 0,05$ ). Al igual que el Grupo 2, este segmento presentó preferencia por ambos alimentos sin MG (valores positivos de preferencia de 0,029 en leche y de 0,056 en salsa de tomate) y rechazo hacia los transgénicos (preferencia negativa de $-0,029$ en leche y de $-0,056$ en salsa

Cuadro 3. Características demográficas, conocimiento de alimentos transgénicos (AT) y lectura de etiquetas con diferencias significativas de grupos identificados mediante análisis de conglomerados en compradores de supermercados de la ciudad de Temuco, Chile. Junio de 2006.

Table 3. Demographic characteristics, knowledge of transgenic modified food and reading of labels with significant differences in the groups of supermarket buyers identified by cluster analysis in Temuco, Chile. June, 2006.

\begin{tabular}{|c|c|c|c|c|c|}
\hline Muestra & $\begin{array}{c}\text { Grupo } 1 \\
\mathrm{n}=42\end{array}$ & $\begin{array}{c}\text { Grupo } 2 \\
\mathrm{n}=187\end{array}$ & $\begin{array}{c}\text { Grupo } 3 \\
n=67\end{array}$ & $\begin{array}{c}\text { Grupo } 4 \\
\mathrm{n}=75\end{array}$ & $\begin{array}{c}\text { Grupo } 5 \\
\mathrm{n}=29\end{array}$ \\
\hline Edad & & & $p=0,0462$ & & \\
\hline$<$ de 35 años & 28,6 & 41,7 & 29,9 & 22,7 & 20,7 \\
\hline $35-54$ años & 54,8 & 39,0 & 46,3 & 54,7 & 44,8 \\
\hline 55 años o más & 16,7 & 19,3 & 23,9 & 22,7 & 34,5 \\
\hline Tamaño del grupo familiar & & & $p=0,0012$ & & \\
\hline 1-2 integrantes & 7,1 & 11,8 & 7,5 & 2,7 & 0,0 \\
\hline 3-4 integrantes & 71,4 & 56,1 & 62,7 & 82,7 & 89,7 \\
\hline 5 ó más & 21,4 & 32,1 & 29,9 & 14,7 & 10,3 \\
\hline Presencia y edad hijos & & & $p=0,0002$ & & \\
\hline Sin hijos & 31,0 & 40,1 & 31,3 & 4,0 & 3,4 \\
\hline Hijos $<5$ años & 2,4 & 9,6 & 4,5 & 14,7 & 10,3 \\
\hline Hijos 5-12 años & 14,3 & 8,0 & 9,0 & 14,7 & 6,9 \\
\hline Hijos $13-17$ años & 31,0 & 19,3 & 35,8 & 48,0 & 41,4 \\
\hline Hijos $\geq 18$ años & 21,4 & 23,0 & 19,4 & 18,7 & 37,9 \\
\hline Estilo de vida & & & $p=0,0262$ & & \\
\hline Conservador & 28,6 & 44,9 & 25,4 & 28,0 & 34,5 \\
\hline Liberal & 35,7 & 29,9 & 43,3 & 41,3 & 48,3 \\
\hline Vegetariano & 9,5 & 11,8 & 3,0 & 16,0 & 10,3 \\
\hline Deportista & 11,9 & 7,5 & 17,9 & 6,7 & 3,4 \\
\hline Innovador & 14,3 & 5,9 & 10,5 & 8,0 & 3,4 \\
\hline Conoce significado AT1 & & & $p=0,0122$ & & \\
\hline $\mathrm{Si}$ & 28,6 & 31,0 & 25,4 & 24,0 & 0,0 \\
\hline No & 71,4 & 69,0 & 74,6 & 76,0 & 100,0 \\
\hline Lectura etiquetas & & & $p=0,0072$ & & \\
\hline Siempre & 19,0 & 10,2 & 7,5 & 12,0 & 27,6 \\
\hline Generalmente & 28,6 & 22,6 & 31,3 & 41,4 & 31,0 \\
\hline Ocasionalmente & 26,2 & 39,2 & 31,3 & 17,3 & 24,1 \\
\hline Casi nunca & 11,9 & 21,0 & 25,4 & 24,0 & 10,3 \\
\hline Nunca & 14,3 & 7,0 & 4,5 & 5,3 & 6,9 \\
\hline
\end{tabular}

${ }^{1}$ AT: alimento transgénico.

${ }^{2}$ Valor p, corresponde a la significancia asintótica (bilateral) obtenida en Prueba $\chi 2$ de Pearson.

${ }^{\prime}$ TF: transgenic food.

${ }^{2} p$ value, is the asymptotic significance (bilateral) obtained in Pearson $\chi 2$ Test. 
de tomate) significativamente inferior al Grupo 2 y similar a los Grupos 1 y $5(\mathrm{p} \leq 0,05)$, pero destacan las bajas magnitudes de preferencia y rechazo. Estos resultados permiten llamar a este segmento "Consumidores sensibles a la marca de los alimentos, rechazan los AT" (Cuadro 2). El Grupo 3 presentó superior proporción de familias numerosas (29,9\%), con hijos entre 13 y 17 años $(35,8 \%)$, de estilo de vida liberal $(43,3 \%)$ y deportista $(17,9 \%)$, y de consumidores que "casi nunca" revisan las etiquetas de los alimentos $(25,4 \%)$ antes de la compra (Cuadro 3).

El cuarto grupo (Grupo 1) representó $(n=42)$ el $10,5 \%$ de la muestra total de encuestados y fue el Grupo que asignó mayor importancia relativa al precio en la compra de leche y salsa de tomate con 59,3 y 53,0\% para leche y salsa de tomate, respectivamente. Fue significativamente superior a los Grupos 2, 3, 4 y $5(\mathrm{p} \leq 0,05)$. Fue mayor la relevancia asignada a la existencia de MG que a la marca, tanto respecto de la leche como de la salsa de tomate ( $20,9 \%$ en leche y $25,7 \%$ en salsa de tomate), pero estadísticamente similar al Grupo 3 en leche y similar a los Grupos 1 y 5 en salsa de tomate $(\mathrm{p} \leq 0,05)$. Al igual que los Grupos 2 y 3 , este grupo evidenció preferencia por los alimentos sin MG (preferencias de 0,006 en leche y 0,196 en salsa de tomate) y rechazo hacia los transgénicos (preferencias de -0,006 en leche y -0,196 en salsa de tomate). Según estos resultados este Grupo se puede llamar "Consumidores sensibles al precio de los alimentos, rechazan los AT" (Cuadro 2). Este grupo presentó mayor proporción de personas entre 35 y 54 años $(54,8 \%)$, de hogares con tres a cuatro integrantes $(71,4 \%)$, con hijos entre 5 y 12 años $(14,3 \%)$, de estilo de vida innovador $(14,3 \%)$ y personas que leen las etiquetas previo a la compra (19\%), pero paralelamente, superior porcentaje de personas que nunca las revisan $(14,3 \%)$ (Cuadro 3).

El grupo minoritario (Grupo 5) representó $(\mathrm{n}=$ 29) el $7,3 \%$ de la muestra total de encuestados y asignó leve mayor importancia al precio en leche $(37,5 \%)$ y a la marca $(39,8 \%)$ en salsa de tomate. La importancia asignada a la existencia de MG fue $32,1 \%$, en leche, situación significativamente inferior a los
Grupos 2 y 4 y superior a los Grupos 1 y 3 ( $\mathrm{p} \leq 0,05), \mathrm{y} 23,1 \%$ en salsa de tomate lo que fue significativamente inferior a los Grupos 2 y 4 y estadísticamente similar a los Grupos 1 y 3 (p $\leq 0,05)$. Al igual que el Grupo 4, este Grupo prefirió los AT (preferencia de 0,017 ) y rechazó ambos alimentos sin MG (preferencia negativa de -0,017) (Cuadro 2). Este grupo presentó mayor proporción de personas de 55 años o más $(34,5 \%)$, de familias con tres a cuatro integrantes $(89,7 \%)$, con hijos entre 13 y 17 años $(41,4 \%)$ y mayores de edad $(37,9 \%)$, estilo de vida liberal $(48,3 \%)$, consumidores que "siempre" revisan las etiquetas de los alimentos $(27,6 \%)$, siendo destacable que el 100\% indicó desconocer previamente el significado de AT (Cuadro 3). De acuerdo con estos resultados este Grupo se puede llamar "Consumidores influenciables con información, prefieren AT".

Independientemente de la importancia asignada a la existencia de MG en los alimentos y de la aceptación o rechazo hacia los AT, los cinco Grupos prefirieron las marcas nacionales (Nestlé y Malloa) y rechazaron la marca propia (cifras negativas de preferencia). La preferencia del Grupo 3 por las marcas nacionales fue significativamente superior al resto de los grupos $(\mathrm{p} \leq 0,05)$. Paralelamente, la mayoría de los grupos identificados presentó similar comportamiento que la muestra total frente a los niveles de precio, a excepción de los Grupos 4 y 5 que presentaron cifras positivas de preferencia ante los niveles de precio en leche y salsa de tomate, respectivamente, las que se incrementaron frente al mayor precio sugiriendo una asociación entre mayor precio y calidad superior.

\section{Discusión}

De acuerdo con los resultados obtenidos, fue posible segmentar la población de Temuco, estableciéndose cinco Grupos en función de la importancia asignada a MG de tipo transgénica en un alimento de origen animal (leche líquida) y otro de origen vegetal (salsa de tomate) y según la actitud de los consumidores hacia los ATs. Al mismo tiempo se determinaron actitudes diferenciales respecto de la presencia de $\mathrm{MG}$ en estos alimentos, demostrando que la respuesta de los consumidores a los ATs no es universal. 
Al mismo tiempo se confirma la percepción positiva de grupos de consumidores argentinos sobre alimentos MG (Mucci y Hough, 2004).

El criterio de segmentación utilizado en este trabajo, basado en el uso de las técnicas multivariadas, análisis conjunto y análisis de conglomerados jerárquicos, fue exitoso y estableció diferencias significativas entre los segmentos obtenidos según el comportamiento de compra de los consumidores de Temuco, Chile (Andrews y Currim, 2000).

En esta investigación, los coeficientes de correlación de Pearson y Tau de Kendall demostraron buena bondad de ajuste del modelo conjunto y la ordenación obtenida correspondió con la ordenación global del encuestado (Hair et $a l .$, 1999). Bajo este contexto, si bien en el total de encuestados la existencia de MG dominó la elección del consumidor, al tener la MG mayor peso relativo que la marca y el precio en la decisión de compra de leche y salsa de tomate (44,7 y 44,6\%; respectivamente), se determinó un comportamiento diferente en tres (Grupos 1, 3 y 5) de cinco segmentos de consumidores.

Para los consumidores de los Grupos 1 y 5 fue más relevante el precio y para los consumidores del Grupo 3 tuvo mayor importancia la marca en la decisión de compra. Asimismo, los valores de preferencia obtenidos para los AT y sin MG en la muestra total, llevarían a concluir que existe preferencia por el alimento tradicional y rechazo hacia el AT (preferencias positivas y negativas, respectivamente). Sin embargo, el análisis segmentado de los consumidores permitió distinguir la existencia de dos segmentos que prefirieron leche y salsa de tomate transgénica (preferencias positivas en los Grupos 4 y 5), aun cuando el segmento más numeroso presentó rechazo por los ATs.

La preferencia por leche y salsa de tomate sin MG y el rechazo hacia los ATs obtenida en la muestra total de encuestados y en particular en los Grupos 1, 2 y 3 (10,5; 46,8 y 16,8\% de la muestra total) concordó con resultados previamente publicados (Ganiere et al., 2006; Ho et al., 2006; Larue et al., 2004; Lusk et al., 2003; Gil et al., 2001; Lusk et al., 2001). No obstante, el comportamiento opuesto de los
Grupos 4 y 5 ( 8,8 y 7,3\% de la muestra total), permite sugerir que en algunos consumidores de Temuco la aceptación de AT se relaciona con los beneficios percibidos y con el conocimiento que el consumidor reciba respecto de este tipo de alimentos (Townsend, 2006; Hossain et al., 2003; Verdume y Viaene, 2003). En efecto, los Grupos 4 y 5 fueron los que presentaron menor proporción de consumidores conocedores del significado de los ATs. Por lo tanto, es posible atribuir la actitud positiva hacia la leche y salsa de tomate transgénica a la información previamente indicada, la que se entregó antes de la presentación de los estímulos desarrollados para el análisis de conjunto.

La proporción de personas con información de AT (59\%) fue superior al 53\% determinado en Brasil (Oda y Soares, 2000). Sin embargo, al considerar que sólo $26,3 \%$ de los encuestados conocía el significado de AT es posible señalar que el conocimiento de la población es aun bajo, situación que concuerda con resultados reportados en EUA (Byrne, 2006) y Santiago, Chile (Gil et al., 2001). En este sentido, la aprobación generalizada de la muestra total $(99,3 \%)$ respecto a incluir en la etiqueta de los alimentos información del uso de ingredientes MG, representa la opción de estar informado para elegir (Anderson y Jackson, 2005; Mucci y Hough, 2004; Napier et al., 2004).

Por otra parte, la existencia de MG fue la variable de decisión de compra más importante en la muestra total de encuestados y en los Grupos 2 y 4 (65,6\% de la muestra total) seguida por la marca y el precio en ambos alimentos. Sin embargo, este atributo tuvo menor importancia en la decisión de compra de los Grupos 1 y 3 en ambos alimentos y del Grupo 5 en salsa de tomate. Esta situación concuerda con lo determinado en EUA, donde el precio fue más importante que la presencia de MG y que la marca del alimento en la decisión de compra de cereales para el desayuno (Baker y Burnham, 2002). No obstante, es importante destacar la similar importancia asignada por los consumidores del Grupo 5 a la marca y a la existencia de MG en leche, indicando que ninguno de estos atributos domina las preferencias del consumidor (Baker y Burnham, 2002). Esto permite señalar que para 
los consumidores de los Grupos 1, 3 y 5 (34,6\% de la muestra total), la existencia de MG no es relevante en la decisión de compra. Además, las cifras de preferencia de los Grupos 1 y 3 hacia ambos alimentos no transgénicos no difirieron estadísticamente del rechazo del Grupo 5, y viceversa respecto de los ATs. Por lo tanto, es posible concluir, tal como se ha reportado previamente, que estos consumidores son indiferentes a la existencia de MG (Townsend, 2006).

Por otra parte, el orden de importancia de la existencia de MG, marca y precio fue similar para leche y salsa de tomate en la muestra total de encuestados y en los cinco Grupos, así como también la aceptación y rechazo hacia los ATs, lo que corrobora que no sólo resulta relevante el consumo directo de ATs sino que también el uso de ATs en alimentación animal (Lähteenmäki et al., 2003; Lusk et al., 2003; Roosen et al., 2003). No obstante, no se observó un mayor rechazo hacia el AT de origen animal (Frewer et al., 2004), posiblemente debido a que la existencia de MG fue relativamente más relevante en salsa de tomate en los Grupos 1, 3 y 4 (46\% de la muestra total), sólo en los Grupos 2 y $5(54 \%)$ este atributo fue más importante en leche pero el rechazo fue mayor frente a salsa transgénica en el Grupo 2, de manera similar a los Grupos 1 y 3. Es decir, estos resultados avalan la existencia de una actitud positiva o negativa generalizada hacia los ATs y no asociada a un producto en particular.

En la muestra total de encuestados y en cada uno los cinco Grupos identificados, la preferencia por las marcas de fabricantes (Nestlé y Malloa) y el rechazo hacia la marca propia se relacionó con el riesgo percibido en la compra de un alimento con marca propia (Sethuraman y Cole, 1999). En forma concordante, los consumidores están más dispuestos a comprar ATs de marcas conocidas (Verdume y Viaene, 2003). No obstante, y desde el punto de vista del riesgo percibido en la elección de compra, fue paradójico que los consumidores de tres Grupos rechazaran los ATs, mientras en los cinco Grupos hubo rechazo hacia la marca propia. Es evidente la necesidad de nuevas investigaciones para precisar estos aspectos.
Por otra parte, la obtención de cinco Grupos de consumidores con una aceptación diferenciada de ATs y con diferentes edades, tamaño del grupo familiar, presencia y edad de los hijos, se contrapone con resultados previos (Hossain y Oyango, 2004; Lusk et al., 2001). En esta investigación se confirma la relación entre la edad del consumidor y la actitud hacia los AT. Sin embargo, el mayor rechazo hacia ATs se obtuvo en el Grupo 2, predominantemente formado por menores de 35 años. En contraposición con estudios previamente publicados, los Grupos que prefirieron ATs presentaron mayor proporción de personas mayores de 35 años (Ganiere et al., 2006; Baker y Burnham, 2002). No obstante, es posible indicar que la superior importancia asignada por el Grupo 2 a la presencia de MG concuerda con la mayor preocupación por el sistema de producción de los alimentos encontrada en consumidores europeos jóvenes (Bernués et al., 2003).

En relación con la conformación del grupo familiar, destaca la presencia y edad de los hijos por su relación con la actitud hacia ATs. Así, mientras los grupos que concentraron mayores porcentajes de hogares con hijos mayores de 13 años (Grupos 4 y 5) presentaron una actitud positiva hacia los ATs. Los Grupos que evidenciaron rechazo presentaron superior proporción de personas sin hijos (Grupos 1, 2 y 3). Esto sugiere que estos posibles futuros padres pondrán gran atención en la compra de alimentos no transgénicos para sus hijos.

A diferencia de la mayor disposición de los hombres a adquirir ATs detectada en EUA y Europa, pero concordante con lo determinado en América del Sur, en el presente estudio los grupos identificados no presentaron diferencias significativas según el género del consumidor $(\mathrm{p}$ $>0,05$ ) (Mucci y Hough, 2004; Hossain et al., 2003; Verdume y Viaene, 2003; Oda y Soares, 2000). Por lo tanto, es posible que se trate de un fenómeno ligado al mercado. De la misma forma, fue posible comprobar la relación entre el estilo de vida del consumidor y la actitud hacia los ATs reportada en EUA (Hossain et al., 2003). Sin embargo, contrariamente a lo reportado por Hossain et al. (2003) en este estudio los Grupos que prefirieron ATs (Grupo 4 y 5) presentaron mayor proporción 
de consumidores autodefinidos con un estilo de vida liberal. El Grupo que claramente rechazó los ATs (Grupo 2) tuvo una elevada proporción de consumidores de estilo de vida conservador.

Considerando que la aceptación de los consumidores hacia los ATs constituye uno de los factores críticos para el uso de biotecnología en la agricultura y en la producción de alimentos, la presente investigación es un aporte al debate mundial sobre la actitud que los consumidores de Temuco tienen hacia los ATs. Este tema ha sido poco investigado en Chile y otros países en vías de desarrollo, en los cuales la biotecnología está presente en la producción de alimentos.

\section{Resumen}

Sobre la base del debate generado por los alimentos genéticamente modificados, particularmente respecto de los alimentos transgénicos (AT), en los países desarrollados, se determinó la importancia de esta variable en la compra de leche líquida y salsa de tomate en consumidores de Temuco (Región de La Araucanía, Chile) y la existencia de diferentes segmentos de mercado, mediante una encuesta a 400 personas. Utilizando análisis conjunto se determinó que la existencia de modificación genética (MG) fue más importante que la marca y el precio en ambos alimentos. Mediante análisis de conglomerados se distinguieron cinco Grupos, dos de ellos con el 46,8 y 18,8\% del total de encuestados. Estos dos Grupos mas numerosos dieron importancia a la existencia de MG, pero sólo el Grupo mayoritario prefirieron las alternativas sin manipulación genética. Para el tercer Grupo $(16,8 \%)$ la marca fue el atributo más relevante, pero mostró predilección por alimentos no transgénicos. El cuarto Grupo $(10,5 \%)$ dio gran importancia al precio y prefirió alimentos no transgénicos. El Grupo minoritario (7,3\%) presentó leve mayor valoración por el precio en leche y por la marca en salsa de tomate, mostrando preferencia por los ATs. Los cinco Grupos rechazan el producto con marca propia y la mayoría reaccionó positivamente frente a menores precios. Por lo tanto, los resultados de esta investigación permiten concluir que la ausencia de MG en leche y salsa de tomate es una condición deseable para una alta proporción de consumidores de Temuco (74\%), pero existen grupos de consumidores (26\%) que aceptan la manipulación genética en estos alimentos.

Palabras clave: Alimentos genéticamente modificados, alimentos transgénicos, análisis de conjunto, análisis de conglomerados, ingeniería genética, mercadeo.

\section{Agradecimientos}

Los resultados presentados se obtuvieron como parte de los trabajos financiados por el Proyecto DIUFRO N 120601 financiado por la Universidad de La Frontera, Temuco, Chile.

\section{Literatura citada}

Anderson, K. y L. Jackson. 2005. GM crop technology and trade restraints: economic implications for Australia and New Zealand. The Australian Journal of Agricultural and Resource Economics 49:263-281.

Andrews, R. y I. Currim. 2003. Recovering and profiling the true segmentation structure in markets: an empirical investigation. International Journal of Research in Marketing 20:177-192.

Anónimo 1. 2002. INIA en el desarrollo de Plantas y otros Organismos Genéticamente Modificados. Instituto Nacional de Investigación Agropecuaria (INIA). Santiago, Chile. www. inia.cl/biotecnologia/publicaciones/GMO_ INIA.pdf. (Consultado: octubre de 2007).

Anónimo 2. 2004. Mapa Socioeconómico de Chile. Adimark, Investigación de Mercados y Opinión Pública. Santiago, Chile. www.adimark.cl (Consultado: abril de 2005).

Baker, G. y T. Burnham. 2002. The market for genetically modified foods: consumer characteristics and policy implications. International Food and Agribusiness Management Review 4:351-360.

Bernués, A., A. Olaizola y K. Corcoran. 2003. Extrinsic attributes of red meat as indicators of quality in Europe: an application for market segmentation. Food Quality and Preference 14:265-276

Byrne, P. 2006. Safety and public acceptance of transgenic products. Crop Science 46:113-117.

Chen, M. y H, Li. 2007. The consumer's attitude toward genetically modified foods in Taiwan. Food Quality and Preference 18:662-674.

Costa-Font, J. y E. Mossialos. 2007. Are perceptions 
of risks and benefits of genetically modified food independent?. Food Quality and Preference 18:173-182.

Fernández, A. 2002. Investigación y Técnicas de Mercado. Editorial Esic. Primera edición. Madrid, España. 273 pp.

Finucane, M.L. y J.L. Holup. 2005. Psychological and cultural factors affecting the perceived risk of genetically modified food: an overview of the literature. Social Science and Medicine 60:1603-1612.

Frewer, L., J. Lassen, B. Kettlitz, J. Scholderer, V. Beekman y K. Berdal. 2004. Societal aspects of genetically modified foods. Food and Chemical Toxicology 42:1181-1193.

Ganiere, P., W. Chern y D. Hahn. 2006. A continuum of consumer attitudes toward genetically modified foods in the United States. Journal of Agricultural Resource Economics 31:129-149.

Gil, L., V. Martínez, C. Irarrázabal y C. Martínez. 2001. Aceptación pública de la biotecnología y de los alimentos transgénicos. Ambiente y Desarrollo (Chile) 17:52-59.

Hair, J., R. Anderson, R. Tatham y W. Black. 1999. Análisis Multivariante. Otero (ed.). Prentice Hall Internacional. Inc. Quinta edición. Madrid, España. $832 \mathrm{pp}$.

Ho, P., E. Vermeer y J. Zhao. 2006. Biotechnology and Food safety in China: Consumers' acceptance or resistance? Development and Change 37:227-253.

Hossain, F., B. Onyango, B. Schilling, W. Hallman y A. Adelaja. 2003. Product attributes consumer benefits and public approval of genetically modified foods. International Journal of Consumer Studies 27:353-365.

Hossain, F. y B. Onyango. 2004. Products attribute and consumer acceptance of nutritionally enhanced genetically modified foods. International Journal of Consumer Studies 28:255-267.

Huang, J., H. Qiu, J. Bai y C. Pray. 2006. Awareness, acceptance of and willingness to buy genetically modified foods in Urban China. Appetite 46:144-151.

Jaeger S, J.L. Lusk, L. House, C. Valli, M. Moore, B. Morrow y B. Traill. 2004. The use of non-hypothetical experimental markets for measuring the acceptance of genetically modified foods. Food Quality and Preference 15:701-714

Kaye-Blake, W., K. Bicknell y C. Saunders. 2005. Process versus product: wish determines consumer demand for genetically modified apples? Australian Journal of Agricultural Resource Economics 49:413.427.

Lähteenmäki, L., K. Grunert, Ø. Ueland, A. Åström, A. Arvola y T. Bech-Larsen. 2003.
Acceptability of genetically modified cheese presented as real product alternative. Food Quality and Preference 13:523-533.

Larue, B., G. West, C. Gendron y R. Lambert. 2004. Consumer response to functional foods produced by conventional, organic, or genetic manipulation. Agribusiness 20:155-166.

Lea, P., M. Rodbotten y T. Naes. 1997. Analysis of variance for sensory data. John Wiley \& Son. First edition. Chichester, United Kingdom. 102 pp.

Lusk, J.L., M. Daniel, D. Mark y C. Lusk. 2001. Alternative calibration and auction institutions for predicting consumer willingness to pay for nongenetically modified corn chips. Journal of Agricultural Resource Economics 26:40-57.

Lusk, J.L., M. Moore, L. House y B. Morrow. 2002. Influence of brand and type of modification on consumer acceptance of genetically engineered corn chips: a preliminary analysis. International Food Agribusiness Management Review 4:320331.

Lusk, J.L., J. Roosen y J.A. Fox. 2003. Demand for beef from cattle administered growth hormones or fed genetically modified cord; a comparison of consumers in France, Germany, the United Kingdom and the United States. American Journal of Agricultural Economics 85:16-29.

Mucci, A. y G. Hough. 2004. Perceptions of genetically modified foods by consumers in Argentina. Food Quality and Preference 15:43-51.

Napier, T., M. Tucker, C. Henry y S. Whaley. 2004. Consumer attitudes toward GMOs: the Ohio experience. Journal of Food Science 69:69-76.

Noussair, C., S. Robin y B. Ruffieux. 2004. Do consumers really refuse to buy genetically modified food? The Economic Journal 114:102-120.

Oda, L.M. y B.E. Soares. 2000. Genetically modified foods: economics aspects and public acceptance in Brazil. Trends in Biotechnology 5:188-190.

Ríos, S. 2005. Cultivos transgénicos en Chile. Observatorio de la Economía Latinoamericana 38:1-14.

Roosen, J., J. Lusk y J. Fox. 2003. Consumer demand for and attitudes toward alternative beef labeling strategies in France, Germany and the UK. Agribusiness 19:77-90.

Sethuraman, R. y C. Cole. 1999. Factors influencing the price premiums that consumers pay for national brands over store brands. Journal of Products and Brand Management 8:340-351.

Townsed, E. 2006. Affective influences on Risk Perceptions of, and attitudes toward genetically modified food. Journal of Risk Research 9:125-139.

Verdume, A. y J. Viaene. 2003. Consumer beliefs and attitude towards genetically modified food: basis for segmentation and implications for communication. Agribusiness 19:91-113. 